\title{
Research of University Examination Database Construction Under New
}

\section{Information Environment}

\author{
Yingjie $\mathrm{FU}^{1, \text { a }}$ \\ ${ }^{1}$ Department of Teaching Affairs, Jilin Agricultural University, 130118, Changchun, China \\ aemail:jlaufyj@126.com
}

Keywords: Examination database construction: Necessity; Innovation

\begin{abstract}
The traditional method for university examination database construction can hardly satisfy university teaching reforms and the development of network educational technology. Specific to the current situation of university examination database construction, the paper expounded the necessity of university examination database construction, and put forward ideas on the reform of university examination database construction.
\end{abstract}

\section{Introduction}

Examination questions setting is an important means to check the teaching quality scientifically, objectively and accurate. And it is an basic method to evaluate the level and competence of the students to master the knowledge. Examination questions setting, test paper composition, examination organization, paper reviewing and scoring etc. are closely interrelated. In case of any defect in any link, the facticity and credibility of teaching quality metrics will be affected. Currently, traditional exam process mainly focus on exam and paper scoring, but ignore the normalization, objectivity and scientificity. Examination questions setting is a weak link of teaching quality assessment; in addition, traditional examination questions setting is subject to high subjectivity which causes significant impact on the facticity and credibility; further more, from the aspect of examination-orientation of students, it is essential for students to take self-examination on the knowledge learned at the class. Students can consolidate the professional knowledge they have learned, and make up for deficiency by taking open simulation tests which is beneficial to improve the teaching quality. To achieve this goal, traditional simulation test is far insufficient. For modern education projects, how to build or design an examination database and test paper generation by utilizing modern technologies is required by the increasing development of modern education.

\section{Necessity of examination database construction}

Examination database construction is the precondition and foundation to realize separation of teaching and examination.

At present, the separation of teaching and examination has become a major direction of teaching reform, through which, the teaching quantity of teachers can be measured and the learning effects of students can be tested more fairly. 1. To establish a perfect examination database creates conditions for the separation of teaching and examination. Therefore, it is the precondition and foundation to realize separation of teaching and examination.

Examination database construction is an important method to promote the teaching quality of teachers.

Examination database construction can eliminate the failure on separation of teaching and 
examination. After taking the solution, teaching and examination organization will be charged by different person which will constitute a restrictive relation between teaching and examination. The lecturers will be get released from examination questions setting so that they should carry out teaching activity strictly in accordance with the provisions specified on teaching outline and exam outline ${ }^{[1]}$. The school shall set up a sound teaching assessment mechanism to evaluate teachers' performance, and formulate corresponding of rewards and punishments approaches which will actively promote the teaching under such an environment of survival of the fittest. Teachers have to input more energy and enthusiasm on teaching and scientific research. In addition, teaching level and teaching ability can be fully reflected and the teaching effects can be significantly improved; also, in order to get higher grades in the exams, the students must work hard instead of making short-term efforts and getting closer with teachers thus to improve students' learning enthusiasm and actually enhance the teaching quality.

The establishment of examination database has provided a communication platform for teachers and students.

Examination database construction is an important part of course construction. On the basis of setting up an examination database and course learning system on campus network, it has provided a communication platform for teachers and students. Teachers can use the platform to update the examination questions, perform online test and on-line mentoring and issue information etc.; students can utilize the platform to raise questions, as well as perform online testing and learning etc.. Teachers can get understand the status of students on grasping the knowledge objectively and accurately via the platform through the exchange of information between teachers and students ${ }^{[2]}$. Furthermore, students can also ask questions specifically and get a reply from the teacher which can avoid the malpractice of traditional "spoon-feeding" teaching, and promote more harmonious and happy “teaching” and "learning” relationship between teachers and students.

Examination database construction can alleviate the burden of teacher.

Traditionally, teachers not only have to select questions from a large number of questions database, but also have to consider the knowledge points, difficulty level and repeat degree in recent times, etc.. It can be easily seen that they are under extremely huge difficulties and labor intensity. The establishment of examination database can facilitate the paper preparation, alleviate their work burden, release them from multifarious labor so that they can put more energy to improve teaching effects and quality.

\section{Realization of the examination database construction}

To improve the construction mechanism of the examination database and to steadily expedite the examination database construction

Examination database construction can be divided into three phases: The first phase is to design a general and intelligent examination database system, namely to build a platform based on the public examination database of campus network; phase II is to disclose the examination database construction platform on the campus network to all the students and teachers of the school to set up examination database for each discipline courses; phase III is to disclose the examination database for each discipline on the campus network to all students and teachers. The first phase is foundational, the second phase is key and the third phase is the purpose ${ }^{[3]}$. The foundation shall be solid, the key phase shall be profound and the purpose lies on applications. Only with an improved examination database mechanism, the examination database construction can be promoted steadily.

To improve the examination database construction at network era is the system safeguard to update the database. In the short term, universities still can adopt project approval mechanism. Step 
I: To optimize the examination database platform construction, perform overall planning, focus on school development efforts to ensure the successful construction of an open test platform, and focus on the versatility and intelligence of examination database; step II: To select one or two basic courses as trail. On the basis of complying with network platform examination database requirements, it shall play the advantages of abundant basic course teaching resources to ensure the qty and quality of examination database; step III: To grasp the project approval link. On the basis of the above mentioned two steps, disclose the examination database to all of the teachers and students to accept test of practice. It has been approved that the examination database is successful. Start up the inspection procedure to ensure absolutely reliable of the inspection; step IV: Gradually promote the mode to other courses by means of project approval based on the open examination database construction. In the medium and long term, perform experience exchange with other universities on examination database construction to further improve database construction thus to realize resource sharing with other universities, and even form disclosed resources of educational network ${ }^{[4]}$.

To adopt new technologies to ensure the generality and intelligence of the open examination database

Whether the open examination database is successful or not depends on three aspects: Firstly, it is necessary to realize the generality of the examination database, namely the system shall be suitable for the examination database construction of each discipline courses; secondly, it shall solve the intelligence of the system and shall instruct and intelligently guide the learning of the students ordinarily; thirdly, a great amount of high-quality examination questions shall be available in the examination database.

Model building technique is adopted by many system development projects. To establish a general model of intelligent test system based on a large number of detailed analysis of the examination database system can link the teachers and students, learning and assessment, as well as teaching and management closely together. The system shall adopt embedded structure. The examination database frame shall be embedded to the system. Subsequently, record the knowledge and examination questions to the database to complete the examination database construction of the displine. The application of model building technique is a reliable guarantee to solve the universality of the examination database.

Intelligent guidance technology can make the appropriate recommendations on learning according to the testing status of each student. The system shall put forward corresponding learning suggestions to friendly constrain the learning progress of the students thus to improve the learning efficiency. The teachers of the course can control the learning progress of students through simple setting with the support of such technology. In addition, the system will not insufficient in flexibility thus to helps students to learn the knowledge more efficiently and realize auxiliary teaching function ${ }^{[5]}$.

Strengthen the teaching and research programs to ensure the inherent quality of the test database. Based on the versatility and intelligence of database system of campus network, whether the technological superiority can be reflected mainly rely on the efficient and abundant curriculum examination database. It means that the relevance between the knowledge and examination question setting quality in the examination database, as well as the quantity and investigated knowledge determines the objectivity and scientific and scientificity. It requires that teachers shall spend considerable time and energy on in depth and detailed researches on the courses, breakdown the knowledge specific to teaching materials and detailed chapters, select a great amount of examination questions according to knowledge points thus to ensure the quality and quantity of the examination database to satisfy different learning degrees for students of all level on the chapters 
and knowledge points. It is worthy to spend time and energy on material collection and preparation. Only grasping the link, the quality and application effects can be ensured.

\section{Conclusion}

The examination database construction shall be based on the school. The teachers shall take the leading role and mobilize the masses of teachers and students to participate in. Greatly expand the scope and depth based on teaching materials. take capacity as the strength and scientific management as the guarantee. The establishment of examination database will provide everlasting resources and services for education through the campus network. It shall be updated, modified or even upgraded along with the educational reform, school development and informational changes thus to constantly improve and get adapt to the latest demands of educational teaching. Therefore, the examination database shall be built to an interactive, digital, intelligent and open sharing resource database to that it will achieve more significant and profound effects on educational teaching.

\section{References}

[1] Wang Xiaoyu. Practice and Thinking on the construction of examination questions bank in Colleges and Universities [J]. Journal of Northeast Agricultural University,2007(04).

[2] Zhai Jiayu. The research and implementation of general examination question database system based on B/S [D]. Beijing University Of Posts And Telecommunications, 2014(2) .

[3] Xie Yuan. The design and implementation of examination question bank management system based on B/S [D]. Xiamen University,2013.

[4] Chen Jianping. Research on the construction of examination questions bank [J]. Economic Research Guide, 2014 (11) .

[5] Jiang Yan. On the construction of the network examination question bank of the University under the separation of teaching and examination [J]. Journal of Social Science of Jiamusi University, 2013 (3). 